\title{
CERTAIN FEATURES OF FUZZY CONTRA-CONTINUOUS FUNCTIONS
}

\author{
M. H. RASHID
}

Department Mathematics, University of Rajshahi, Rajshahi-6205, Bangladesh.

AND

D.M. ALI

Department of Mathematics, University of Rajshahi-6205, Bangladesh.

\begin{abstract}
We deal with fuzzy topological spaces, fuzzy compact space, fuzzy S-closed space, fuzzy graph, fuzzy continuous functions and fuzzy LC-continuous functions. In this paper, we introduce the concepts of fuzzy contra-continuities and explore properties and relationships of such types of functions.
\end{abstract}

Keywords: fuzzy contra-continuity, fuzzy S-closed space, fuzzy graph.

AMS Subject Classification: 54A40.

\section{INTRODUCTION}

C L. Chang ${ }^{(1)}$ defined fuzzy topological space in 1968 by using fuzzy sets introduced by Zadeh. In 1976, Thompson ${ }^{(2)}$ has introduced the notion of S-closed spaces via Levine's semi-open sets ${ }^{(3)}$. In 1981 Azad $^{(4)}$ has introduced some weaker forms of fuzzy continuity in fuzzy topological spaces. He introduced fuzzy semi-continuous functions, semi-open functions, semi-closed functions, almost continuous functions and weakly continuous functions in fuzzy topological spaces. Using Azad's notion of fuzzy sets Abdulla and Bin Shahna ${ }^{(5)}$ have introduced fuzzy $\delta$-open, fuzzy $\delta$-closed, fuzzy pre-open, fuzzy pre-closed sets and have made preliminary study of fuzzy strong semi-continuous and fuzzy pre-continuous functions in their papers. In 1989, Ganster and Reilly ${ }^{(6)}$ introduced the notion of LC-continuous functions via the concepts of locally closed sets. In 1996, Dontchev ${ }^{(7)}$ studied a stronger form of LC-continuity called contra-continuity and proved that contra-continuous images of strongly S-closed spaces are compact as well as that contra-continuous, $\rho$-continuous images of S-closed spaces are also compact. In 2006, Ekici and Kerre ${ }^{(8)}$ studied the notion of fuzzy contra- $\rho$-continuous functions. In our study, we introduce several types of fuzzy contra-continuities, the notion of fuzzy contra-semi-continuous functions, fuzzy contra-pre-continuous functions and investigate of some of their properties.

\section{Preliminaries}

In this section, we recall some definitions and some results, which will be useful in our investigations. 
2.1 Definition: Let $X$ be a non-empty set and $I=[0,1]$. A fuzzy set in $X$ is a function $\lambda: X \rightarrow I$ which assigns to each element $x \in X$, a degree or grade of membership $\lambda(x) \in I$. Fuzzy sets in $\mathrm{X}$ will be denoted by Greek letters as $\alpha, \beta, \lambda, \mu$, $\eta$, etc.

2.2 Definition ${ }^{(1)}$ : Let $\mathrm{X}$ be a non-empty set and $t$ be a collection of fuzzy sets in $\mathrm{X}$. Then $t$ is called a fuzzy topology in $\mathrm{X}$ if.

(i) $0,1 \in t$

(ii) $\alpha, \beta \in t \Rightarrow \alpha \cap \beta \in t$ and (iii) $\alpha_{\mathrm{i}} \in t \Rightarrow \psi \alpha_{\mathrm{i}} \in t$.

Then the pair $(X, t)$ is called a fuzzy topological space (in brief fts). Every member of $t$ is called a fuzzy open set.

2.3 Definition: Let $\lambda$ be a fuzzy set in $\mathrm{fts}(X, t)$. Then the closure of $\lambda$ is denoted by $\operatorname{cl}(\lambda)$, is given by $\operatorname{cl}(\lambda)=\cap\left\{\mu: \lambda \subseteq \mu\right.$ and $\left.\mu \in t^{\mathrm{c}}\right\}$ and the interior of $\lambda$ is denoted by $\operatorname{int}(\lambda)$, is given by $\operatorname{int}(\lambda)=\cup\{\mu: \mu \subseteq \lambda$ and $\mu \in t\}$.

2.4 Definition ${ }^{(9)}$ : A fuzzy singleton in $X$ is a fuzzy set in $X$ which is zero everywhere, except at one point, say $x$, where it takes value, say $\mathrm{r}$, with $\mathrm{r} \in(0,1]$ i.e. $0<$ $\mathrm{r} \leq 1$. We denote it by $x_{r}$, where the point $x$ is called its support and $r$ its value. Also $x_{r} \in \alpha$ if and only if $\mathrm{r} \leq \alpha(x)$.

2.5. Definition : A fuzzy singleton $x_{r}$ is called quasi-coincident (in short qcoincident) with a fuzzy set $a$ in X, denoted $x_{r} q a$ iff $r+a(\mathrm{x})>1$. Similarly, a fuzzy set $a$ in $\mathrm{X}$ is called q-coincident with a fuzzy set $\beta$ in X. denoted $\alpha q \beta$ iff $\alpha(\mathrm{x})+\beta(\mathrm{x})>1$, for some $\mathrm{x} \in X$.

2.6. Definition ${ }^{(4)}$ : Let $(X, \mathrm{t})$ and $(\mathrm{Y}, \mathrm{s})$ be two fuzzy topological spaces and let $f: \mathrm{X}$ $\rightarrow \mathrm{Y}$ be function between them. Then the function $\mathrm{g}: \mathrm{X} \rightarrow \mathrm{X} \times \mathrm{Y}$ defined by $\mathrm{g}\left(x_{r}\right)=\left(x_{r}, f\right.$ $\left(x_{r}\right)$ is called the fuzzy graph of $f$.

2.7. Definition ${ }^{(4)}$ : A fuzzy set $\alpha$ in an fts $(X, \mathrm{t})$ is called fuzzy semi-open if $a \subseteq \mathrm{cl}($ int $(\alpha)$ ). The complement of a fuzzy semi-open set is said to be fuzzy semi-closed.

2.8. Definition ${ }^{(5)}$ : A fuzzy set $\alpha$ in fts $(X, \mathrm{t})$ is called fuzzy pre-open iff $\alpha \subseteq$ $\operatorname{int}(\operatorname{cl}(\alpha))$. The complement of a fuzzy pre-open set is said to be fuzzy pre-closed.

2.9. Definition ${ }^{(10,11)}$ : A fuzzy set $\alpha$ in its fts $(X, \mathrm{t})$ is called fuzzy semi-pre-open or $p$ open iff $\alpha \subseteq \operatorname{cl}(\operatorname{int}(\operatorname{cl}(\alpha))$. The complement of a fuzzy semi-pre-open set is said to be fuzzy semi-pre-closed or fuzzy $\rho$-closed.

2.10. Definition ${ }^{(8)}$ : Let $\mu$ be a fuzzy set in fts $(X, \mathrm{t})$. The fuzzy $\rho$-closure and $\rho$ interior of $\mu$, denoted by $\rho$-cl $(\mu)$ and $\rho$-int $(\mu)$ are defined by $\Lambda\{\lambda: \mu \subseteq \lambda, \lambda$ is $\rho$ closed $\}$ and $\vee\{\lambda: \mu \supseteq \lambda, \lambda$ is $\rho$ - open $\}$ respectively.

2.11. Definition ${ }^{(8)}$ : Let $\mathrm{X}$ and $\mathrm{Y}$ be fuzzy topological spaces. A function $f: \mathrm{X} \rightarrow \mathrm{Y}$ is said to be fuzzy contra- $\rho$-continuous if for each fuzzy singleton $x_{r} \in X$ and each fuzzy closed set $\mu$ in Y containing $f\left(x_{r}\right)$, there exists a fuzzy $\rho$-open set $\lambda$ in X containing $x_{r}$ such that $\int(\lambda) \subseteq \mu$

3. Fuzzy contra-semi-continuous functions 
In this section, we introduce several types of fuzzy contra-continuous functions and characterize the fuzzy contra-semi-continuous functions in particular.

3.1. Definition: Let $X$ and $Y$ be fuzzy topological spaces. A function $f: X \rightarrow Y$ is said to be fuzzy contra-continuous if for each fuzzy singleton $x_{r} \in \mathrm{X}$ and each fuzzy closed set $\mu$ in Y containing $f\left(x_{r}\right)$, there exists a fuzzy open set $\lambda$ in $\mathrm{X}$ containing $x_{r}$ such that $f(\lambda) \subseteq \mu$.

3.2. Definition: Let $X$ and $Y$ be fuzzy topological spaces. A function $f: X \rightarrow Y$ is said to be fuzzy contra-semi-continuous if for each fuzzy singleton $x_{r} \in X$ and each fuzzy closed set $\mu$ in Y containing $f\left(x_{r}\right)$, there exists a fuzzy semi-open set $\lambda$ in X containing $x_{r}$ such that $f(\lambda) \subseteq \mu$.

3.3. Definition: Let $X$ and $Y$ be fuzzy topological spaces. A function $f: X \rightarrow Y$ is said to be fuzzy contra-pre-continuous if for each fuzzy singleton $x_{r} \in X$ and each fuzzy closed set $\mu$ in Y containing $f\left(x_{r}\right)$, there exists a fuzzy pre-open set $\lambda$ in $\mathrm{X}$ containing $x_{r}$ such that $f(\lambda) \subseteq \mu$.

3.4. Theorem : Let $(X, t)$ and $(Y, s)$ be fuzzy topological spaces and let $f: X \rightarrow Y$ be a function. Then the following statements are equivalent.

(1) $f$ is fuzzy contra-semi-continuous function.

(2) For every fuzzy closed $\mu$ in $Y, f^{1}(\mu)$ is fuzzy semi-open in $\mathrm{X}$,

(3) For every fuzzy open set $\lambda$ in $\mathrm{Y}, f^{1}(\lambda)$ is fuzzy semi-closed in $\mathrm{X}$.

Proof : (1) $\Leftrightarrow$ (2): Let $\alpha$ be fuzzy closed set in $Y$ and let $x_{r} \in f^{1}(\alpha)$. Since $f\left(x_{r}\right) \in \alpha$, by (1), there exists a fuzzy semi-open set $\mu x_{r}$ in X containing $x_{r}$ such that $f\left(\mu x_{r}\right) \subseteq \alpha \Rightarrow$ $\mu x \subseteq f^{1}(\alpha)$. Therefore, $f^{1}(\alpha)$ is fuzzy semi-open, which proves (2).

Conversely, let $x_{r} \in X$ and $\mu$ be a fuzzy closed set in $Y$ containing $f\left(x_{r}\right)$. Then by (2), $f^{1}(\mu)$ is fuzzy semi-open. Put $\lambda=f^{1}(\mu)$. Then $\lambda$ is a fuzzy open set in X containing $x_{\mathrm{r}}$ and hence $f(\lambda) \subseteq \mu$. This shows that $f$ is fuzzy contra-semi-continuous function.

(2) $\Leftrightarrow$ (3) Let $\lambda$ is a fuzzy open set in Y. Put $\mu=\lambda^{c}$. Then $\mu$ is a fuzzy closed set in $Y$. Then by (2), $f^{1}(\mu)$ is fuzzy semi-open. Now, $f^{1}(\mu)=f^{1}\left(\lambda^{\mathrm{c}}\right) \Rightarrow f^{1}(\mu)=\left(f^{1}(\lambda)\right)^{\mathrm{c}}$

$\left.\Rightarrow f^{1}(\mu)\right)^{\mathrm{c}}=$. This implies that $\left(f^{1}(\lambda)\right.$ is fuzzy semi-closed, which is (3). The converse is similar.

3.5 Theorem: Let $(X, t)$ and $Y, s)$ be fuzzy topological spaces and let $f: X \rightarrow Y$ be a function. Then the following statements are equivalent:

(1) For any fuzzy closed set $\mu$ in $Y$ and for any $x_{r} \in X, f\left(x_{r}\right) q \mu$ implies that $x_{r} q$ s-int $\left(f^{1}(\mu)\right)$.

(2) For any fuzzy closed set $\mu$ in $\mathrm{Y}$ and for any $x_{r} \in X$, if $f\left(x_{r}\right) q \mu$, there exists a fuzzy semi-open set $\lambda$ such that $x_{r} q \lambda$ and $f(\lambda) \subseteq \mu$.

Proof : Suppose (1) is true. Let $\mu$ be fuzzy closed set in $Y$ and let $f\left(x_{r}\right) q \mu$, for any $x_{r}$ $\in X$. Then by (1), we have $x_{r} q$ s-int $\left(f^{1}(\mu)\right)$. Put $\lambda=$ s-int $\left(f^{1}(\mu)\right)$, then $f(\lambda)=f\left(\operatorname{sint}\left(f^{1}(\mu)\right)\right)$ $\Rightarrow f(\lambda) \subseteq f\left(f^{1}(\mu)\right) \subseteq \mu$, which proves $(2)$. 
Conversely, suppose that (2) is true. Let $\mu$ be fuzzy closed set in Y and let $f\left(x_{r}\right) q \mu$, for any $x^{r} \in X$. Then by (2), there exists a fuzzy semi-open set $\lambda$ such that $x_{r} q \lambda$ and $\mathrm{f}(\lambda)$ $\subseteq \mu$. This implies that $\mu \supseteq f\left(f^{-1}(\mu)\right)$, $f\left(\right.$ s-int $\left(f^{-1}(\mu)\right)=f(\lambda) \Rightarrow \lambda=$ s-int $\left(f^{-1}(\mu)\right)$. Hence, $x_{r}$ q s-int $\left(f^{-1}(\mu)\right)$, which proves (1).

3.6. Definition: Let $(X, \mathrm{t})$ and $(Y, s)$ be two fuzzy topological spaces. A function $f: X$ $\rightarrow \mathrm{Y}$ is called a fuzzy s-irresolute if the inverse image of each fuzzy semi-open if the direct image of each fuzzy semi-open set is fuzzy semi-open.

3.7. Definition: Let $(X, t)$ and $(Y, s)$ be two fuzzy topological spaces. A function $f: X$ $\rightarrow Y$ is called a fuzzy semi-open if the direct image of each fuzzy semi-open set is fuzzy semi-open.

3.8. Theorem: Let $(X, t),(Y, s)$ and $(Z, u)$ be fuzzy topological spaces and let $f: X \rightarrow Y$ and $\mathrm{g}: Y \rightarrow Z$ be functions. If $f$ is fuzzy s-irresolute and $g$ is fuzzy contra-semicontinuous, then $g^{\circ} f$ is fuzzy contra-semi-continuous function.

Proof: Let $\mu$ be a fuzzy closed set in $Z$ and let $\left(g{ }^{\circ} f\right)\left(x_{r}\right) \in \mu$, for every fuzzy singleton $x^{r}$ in $\mathrm{X}$. Then, we have $\mathrm{g}\left(f\left(x_{r}\right)\right) \in \mu$. Since $\mathrm{g}$ is fuzzy contra-semi-continuous, there exists a fuzzy semi-open set $\lambda$ containing $f\left(x_{r}\right)$ such that $g(\lambda) \subseteq \mu$. Again, since $f$ is fuzzy s-irresolute, there exists a fuzzy semi-open set $\eta$ containing $x_{r}$ such that $f(\eta) \subseteq \lambda$. Hence, we have $\left(\mathrm{g}^{\circ} f\right)(\eta)=\mathrm{g}(f(\eta)) \subseteq \mathrm{g}(\lambda) \Rightarrow\left(\mathrm{g}^{\circ} f\right)(\eta) \subseteq \mu$. This shows that $g^{\circ} f$ is fuzzy contra-semi-continuous function. This completes the proof of the theorem,

3.9. Theorem: Let $(X, t),(Y, s)$ and $(Z, u)$ be fuzzy topological spaces. If $f: X \rightarrow Y$ is a surjective fuzzy semi-open function and $g: Y \rightarrow Z$ is a function such that $g{ }^{\circ} f$ is fuzzy contra-semi-continuous, then $g$ is fuzzy contra-semi-continuous.

Proof: Let $\mu$ be a fuzzy closed set in $Z$ let $\left(g^{\circ} f\right)\left(x_{r}\right) \in \mu$, for every fuzzy singleton $x_{r}$ $X$. Then, we have $g\left(f\left(x_{r}\right)\right) \in \mu$. Since $g{ }^{\circ} f$ is fuzzy contra-semi-continuous, there exists a fuzzy semi-open set $\lambda$ in $X$ containing $x_{r}$ such that $g(f(\lambda)) \subseteq \mu$. Again since $f$ is a surjective fuzzy semi-open, $f(\lambda)$ is a semi-open set in Y containing $f\left(x_{r}\right)$ such $g(f(\lambda)) \subseteq \mu$. This shows that $g$ is fuzzy contra-semi-continuous function. This completes the proof of the theorem.

3.10. Theorem: Let $(X, t)(Y, s)$ and $(Z, u)$ be fuzzy topological spaces and let $\mathrm{f}: X \rightarrow Y$ and $g: Y \rightarrow Z$ be functions. If $f$ is fuzzy continuous and $g$ is fuzzy contra-semi-continuous, then $g^{\circ} f$ is fuzzy contra-semi-continuous function.

Proof: Let $\mu$ be a fuzzy closed set in $Z$ and let $\left.g^{\circ} f\right)\left(x_{r}\right) \in \mu$, for every fuzzy singleton $x_{r}$ in $\mathrm{X}$. Then, we we have $\mathrm{g}\left(f\left(x_{r}\right)\right) \in \mu$. Since $g$ is fuzzy contra-semi-continuous, there exists a fuzzy open set $\lambda$ in Y containing $f\left(x_{r}\right)$ such that $g(\lambda) \subseteq \mu$. Again, since $f$ is a fuzzy continuous, there exists a fuzzy open set $\eta$ in $\mathrm{X}$ containing $x_{r}$ such that $\subseteq \int^{-1}(\lambda) \Rightarrow \int(\eta) \subseteq \lambda$. Now, we have $\left(g^{\circ} f\right)(\eta)=\mathrm{g}(f(\eta)) \subseteq g(\lambda) \subseteq \subseteq \mu$, so that $\mathrm{g}^{\circ} f$ is fuzzy contra-semi-continuous.

3.11. Theorem: Let $f: X \rightarrow Y$ be a function and let $g: X \rightarrow X \times Y$ be the fuzzy graph of $f$, defined by $g\left(x_{r}\right)=\left(x_{r}, f\left(x_{r}\right)\right)$ for every $x_{r} \in X$. If $g$ is fuzzy contra-semi-continuous, then $f$ is fuzzy contra-semi-continuous. 
Proof: Let $\mu$ be a fuzzy closed set in Y containing $f\left(x_{r}\right)$ for every $x_{r} \in X$. Then $\mathrm{X} x \mu$ is a fuzzy closed set in $X \times Y$ and $f^{-1}(\mu)=g^{-1}(X \times \mu)$. Since $g$ is fuzzy contra-semicontinuous, then there exists a fuzzy semi-open set $\lambda$ in $X$ containing $x_{r}$ such that $g(\lambda) \subseteq$ $X \times \mu$. This implies that $\lambda \subseteq g^{-1}(X \times \mu)$. Thus, we have $\lambda \subseteq f^{-1}(\mu) \Rightarrow \int(\lambda) \subseteq \mu$. It follows that $f$ is fuzzy contra-semi-continuous function.

\section{Properties of fuzzy contra-continuous functions}

In this section, we investigate the properties and preservation theorems of fuzzy contra-semi-continuous function.

4.1. Definition: Let $(X, t)$ be a fuzzy topological space. Then the fuzzy topological space $(X, t)$ is said to be fuzzy s-compact if every fuzzy semi-open cover of $\mathrm{X}$ has a finite subcover.

4.2. Definition: An fts $(X, t)$ is said to be fuzzy strongly s-closed if every fuzzy semiclosed cover of $\mathrm{X}$ has finite subcover.

4.3. Definition: An fts $(X, t)$ is said to be fuzzy strong countably s-closed if every fuzzy countable semi-closed cover of $X$ has a finite subcover.

4.4. Definition: An fts $(X, t)$ is said to be fuzzy countably s-compact if every fuzzy countable semi-open cover of $X$ has a finite subcover.

4.5. Definition: An fts $(X, t)$ is said to be fuzzy s-Lindelöf if every fuzzy semi-open cover of $X$ has a finite countable subcover.

4.6. Definition: An fts $(X, t)$ is said to be fuzzy strongly s-Lindelof if every fuzzy semi-closed cover of $X$ has a finite countable subcover.

4.7. Theorem: Let $(X, t)$ be a fuzzy s-compact space. If $f: X \rightarrow Y$ is a surjective fuzzy contra-semi-continuous, then the image of $f$ is fuzzy strongly s-closed space.

Proof: Let $\left\{\mu_{i}: i \in I\right\}$ be any fuzzy closed cover of Y. Since $f$ is fuzzy contra-semicontinuous, there exists a fuzzy semi-open set $\left\{\mathrm{f}^{-1}\left(\mu_{i}\right): i \in I\right\}$ which is a fuzzy semi-open cover of $X$. Again, since $(X, \mathrm{t})$ is a fuzzy s-compact space, there exists a finite subset $I_{0}$ of $I$ such that $\mathrm{X}=\mathrm{V}\left\{f^{-1}\left(\mu_{i}\right): i \in I_{0}\right\}$. It follows that $f(X)=\mathrm{V}\left\{\mu_{i}: i \in I_{0}\right\}$. Since $f$ is surjective, then we have $\left.Y=V\left\{\mu_{i}\right): i \in I_{0}\right\}$ and therefore the image if $f$ is fuzzy strongly sclosed. This competes the proof of the theorem.

4.8 Theorem: Let $(X, t)$ be a fuzzy countably s-compact space. If $f: X \rightarrow Y$ is a surjective fuzzy contra-semi-continuous function, then the image of $f$ is fuzzy strong countably s-closed space.

The proof of this theorem can be obtained following the proof of Theorem ([4.7]).

4.9. Theorem: Let $(X, t)$ be a fuzzy s-Lindelof space. If $f: X \rightarrow Y$ is surjective fuzzy contra-semi-continuous, then the image of $f$ is fuzzy strongly s-Lindelof space.

The proof is similar to that of Theorem ([4.7]).

4.10. Definition ${ }^{(12)}$ : A fts $(X, t)$ is called fuzzy connected if $X$ is not the union of two disjoint non-empty fuzzy open sets.

4.11. Definition : A fts $(X, t)$ is called fuzzy s-connected if $X$ is not the union of two disjoint non-empty fuzzy semi-open sets. 
4.12. Theorem: Let $(X, t)$ - and $(Y, s)$ be two fuzzy topological spaces. If $f: X \rightarrow Y$ is a subjective fuzzy contra-semi-continuous function and $X$ is fuzzy s-connected, then $Y$ is fuzzy connected.

Proof: Suppose $Y$ is not a fuzzy connected space. Then there exists non-empty disjoint fuzzy open sets $\mu_{1}$ and $\mu_{2}$ such that $Y=\mu_{1} v \mu_{2}$. Therefore, $\mu_{1}$ and $\mu_{2}$ are fuzzy clopen in $Y$. Since $f$ is fuzzy contra-semi-continuous and onto, then $f^{-1}\left(\mu_{1}\right)$ and $f^{-1}\left(\mu_{2}\right)$ are fuzzy semi-open in $X$. Moreover, $f^{-1}\left(\mu_{1}\right)$ and $f^{-1}\left(\mu_{2}\right)$ are non-empty disjoint and $X=$ $f^{-1}\left(\mu_{1}\right) \vee f^{-1}\left(\mu_{2}\right)$. This shows that $X$ is not fuzzy s-connected, which contradicts our assumption. Therefore, $\mathrm{Y}$ is fuzzy connected.

4.13. Definition: Let $(X, t)$ be a fuzzy topological space. An fts $(X, t)$ is called a fuzzy s-ultra-connected if every pair of non-empty fuzzy semi-closed subsets of $X$ intersects.

4.14. Definition ${ }^{(8)}$ : Let $(X, t)$ be a fuzzy topological space. An $\mathrm{fts}(X, t)$ is called fuzzy hyper-connected if every fuzzy open set is dense.

4.15. Theorem: Let $(X, t)$ and $(Y, s)$ be two fuzzy topological spaces. If $f: X \rightarrow Y$ is a surjective fuzzy contra-continuous function and $X$ is fuzzy s-ultra-connected, then $Y$ is fuzzy hyper-connected.

Proof: Suppose $Y$ is not a fuzzy hyper-connected space. Then there exists a fuzzy open set $\mu$ such that $\mu$ is not dense in $Y$. Therefore, there exists non-empty fuzzy semiopen subsets $\mu_{1}$ and $\mu_{2}$ in $Y$. Since $f$ is fuzzy contra-semi-continuous, then by

Theorem ([3.4]) we can write $\lambda_{1}=f^{-1}\left(\mu_{1}\right)$ and $\lambda_{2}=f^{-1}\left(\mu_{2}\right)$ are disjoint non-empty fuzzy semi-closed sets in $X$, which contradicts the fact that $\lambda_{1}$ and $\lambda_{2}$ interesect i.e. $X$ is fuzzy s-ultra-connected. Therefore, we conclude that $Y$ is fuzzy hyper-connected.

\section{REFERENCES}

1. C. L. Chang, “Fuzzy Topological Spaces”, J. Math. Anal. Appl., 24, 182-192, 1968.

2. T. Thompson, S-closed Spaces, Proc. Amer. Math. Soc., 60, 335-338, 1976.

3. N. Levine, "Semi-open Sets and Semi-continuity in Topological Spaces," Amer. Math. Monthly, 70, 36-41, 1963.

4. K. K. AzAd "On Fuzzy Semi-Continuity, Fuzzy Almost Continuity and Fuzzy Weakly Continuity,” J. Math Anal. Appl., 82, 14-32, 1981.

5. S. Abdulla Bin Shahna, “On Fuzzy Strong Semi-Continuity and fuzzy pre-Continuity,” Fuzzy Sets and Systems, 44, 303-308, 1991.

6. M. GANSTeR AND I. L. ReILly "Locally Closed Sets and LC-continuous functions” Internat. J. Math. Math. Sci. 3, 417-424, 1989.

7. J. Dontchev. "Contra-Continuous Functions and Strongly S-closed spaces. Internat. J. Math. Math. Sci., 2, 303-310, 1996.

8. E. EkICI E. E. Kerre, “On Fuzzy contra-Continuities”, Adv. Fuzzy Math., 1, 35-44, 2006.

9. P. PaO-Ming and L., Ying-Ming "Fuzzy Topology I. Neighborhood Structure of a Fuzzy Point and Moore-Smith Convergence,' J. Math. Anal. Appl., 76, 571-599, 1980. 
10. A. S. Mashhour, M. H. Ghaim and M. A. Fath Alla "On Fuzzy Non-continuous Mappings,” Bull. Cal. Math. Soc., 78, 57-69, 1986.

11. S. S. ThaKuR AND S. Singh, "Fuzzy Semi-preopen Sets and Fuzzy Semi-precontinuity," Fuzzy Sets and Systems, 98, 383-391, 1998.

12. K. S. Raja Sethupathy and S. Laksmivarahan "Connectedness in Fuzzy Topology", Kybernetika 13(3), 190-193, 1977.

Journal of Bangladesh Academy of Sciences, Vol. 32, No. 1, 71-77, 2008 